\title{
Plasma cells promote osteoclastogenesis and periarticular bone loss in autoimmune arthritis
}

\author{
Noriko Komatsu, ${ }^{1}$ Stephanie Win, ${ }^{1}$ Minglu Yan, ${ }^{1}$ Nam Cong-Nhat Huynh, ${ }^{1}$ Shinichiro Sawa, ${ }^{2}$ Masayuki Tsukasaki, \\ Asuka Terashima, ${ }^{3}$ Warunee Pluemsakunthai, ${ }^{1}$ George Kollias, ${ }^{4,5}$ Tomoki Nakashima, ${ }^{6}$ and Hiroshi Takayanagi' \\ 'Department of Immunology, Graduate School of Medicine and Faculty of Medicine, The University of Tokyo, Tokyo, Japan. ²Division of Mucosal Immunology, Research Center for Systems Immunology, \\ Kyushu University, Fukuoka, Japan. ${ }^{3}$ Department of Osteoimmunology, Graduate School of Medicine and Faculty of Medicine, The University of Tokyo, Tokyo, Japan. ${ }^{4}$ Biomedical Sciences Research Centre \\ "Alexander Fleming," Vari, Greece. ${ }^{5}$ Department of Physiology, Medical School, National and Kapodistrian University of Athens, Athens, Greece. ${ }^{6}$ Department of Cell Signaling, Graduate School of Medical and \\ Dental Sciences, Tokyo Medical and Dental University, Tokyo, Japan.
}

\begin{abstract}
In rheumatoid arthritis (RA), osteoclastic bone resorption causes structural joint damage as well as periarticular and systemic bone loss. Periarticular bone loss is one of the earliest indices of RA, often preceding the onset of clinical symptoms via largely unknown mechanisms. Excessive osteoclastogenesis induced by receptor activator of NF- $\kappa B$ ligand (RANKL) expressed by synovial fibroblasts causes joint erosion, whereas the role of RANKL expressed by lymphocytes in various types of bone damage has yet to be elucidated. In the bone marrow of arthritic mice, we found an increase in the number of RANKL-expressing plasma cells, which displayed an ability to induce osteoclastogenesis in vitro. Genetic ablation of RANKL in B-lineage cells resulted in amelioration of periarticular bone loss, but not of articular erosion or systemic bone loss, in autoimmune arthritis. We also show conclusive evidence for the critical contribution of synovial fibroblast RANKL to joint erosion in collagen-induced arthritis on the arthritogenic DBA/1j background. This study highlights the importance of plasmacell RANKL in periarticular bone loss in arthritis and provides mechanistic insight into the early manifestation of bone lesion induced by autoimmunity.
\end{abstract}

\section{Introduction}

Rheumatoid arthritis (RA) is an autoimmune disease characterized by chronic joint inflammation along with bone damage such as localized bone erosion, periarticular bone loss, and systemic bone loss (1-3). The autoimmune process in RA starts with the presentation of autoantigen to $\mathrm{T}$ cells, which help $\mathrm{B}$ cells to differentiate into plasma cells that produce autoantibodies, such as anti-citrullinated protein antibodies (ACPAs) and rheumatoid factors (1-3). Activated $\mathrm{T}$ cells also stimulate synovial inflammation and induce synovial fibroblasts to express receptor activator of NF- $\mathrm{BB}$ ligand (RANKL) through interleukin 17 (IL-17) and other proinflammatory cytokines (3-5). RANKL is the key contributor to enhanced osteoclastogenesis that causes bone destruction in the joints $(6,7)$. Autoantibodies and immune complexes promote bone erosion through FcR $\gamma$ signaling in osteoclast precursor cells or innate immune cells $(8,9)$.

Biologic and targeted synthetic disease-modifying antirheumatic drugs (DMARDs) are quite effective in controlling inflammation and localized bone erosion (1), but little evidence has been obtained about their effect on periarticular and/or systemic bone loss $(10,11)$, which is associated with the impaired physical func-

Conflict of interest: The Department of Osteoimmunology is an endowed department, supported by unrestricted grants from AYUMI Pharmaceutical, Chugai Pharmaceutical, MIKI HOUSE, and Noevir.

Copyright: () 2021, American Society for Clinical Investigation.

Submitted: August 14, 2020; Accepted: January 27, 2021; Published: March 15, 2021

Reference information: J Clin Invest. 2021;131(6):e143060.

https://doi.org/10.1172/JCl143060 tion and high ratio of fractures observed in RA patients (12). Periarticular bone loss begins in the earliest stage of the disease progression of RA, even observed several years before the clinically evident onset (12-14), but the cells and/or molecules involved in periarticular bone loss are at present only poorly understood.

Periarticular bone loss occurs in the bone marrow near inflamed joints. This is partly because of a relatively high concentration of proinflammatory cytokines induced in synovium (13, $15,16)$. The bone marrow is the site where long-lived plasma cells reside (17-19) and it is well documented that ACPAs are observed prior to the clinical onset of RA, suggesting that the plasma cells may contribute to periarticular bone loss through autoantibody production $(1,2,13,15)$. However, it remains unclear which types of cells produce RANKL and thus critically contribute to the periarticular bone loss. Here, we identified a role for bone marrow plasma cells as a RANKL-expressing osteoclastogenic cell subset in periarticular bone loss.

\section{Results and Discussion}

It is critical to determine the RANKL-expressing cells under specific pathologic conditions, but there are limitations to the use of the currently available anti-RANKL antibodies, which are not always suitable for detecting RANKL (encoded by Tnfsf11) expression in certain cell types such as freshly isolated bone marrow cells (Supplemental Figure 1; supplemental material available online with this article; https://doi.org/10.1172/ JCI143060DS1). Thus, we newly generated RANKL-Cre mice, in which Cre recombinase is driven by the Tnfsf 11 promoter 
A
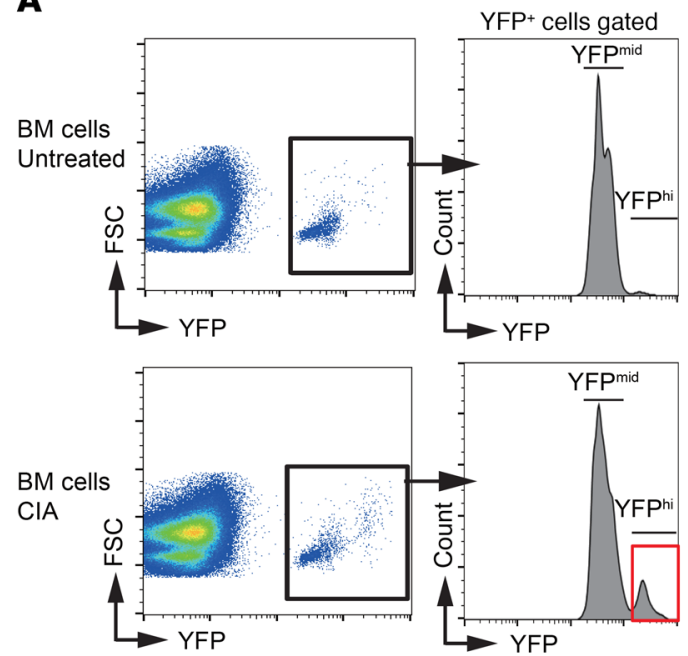

B
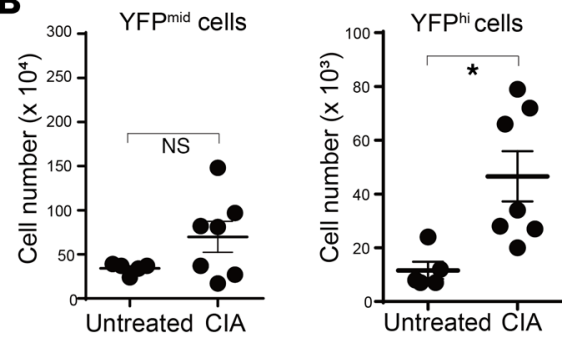

D

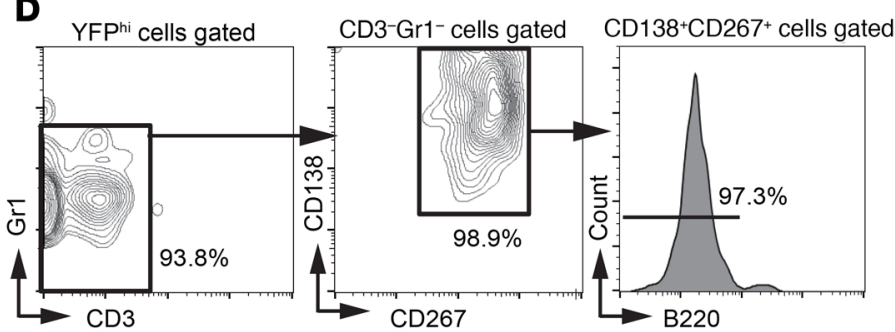

C

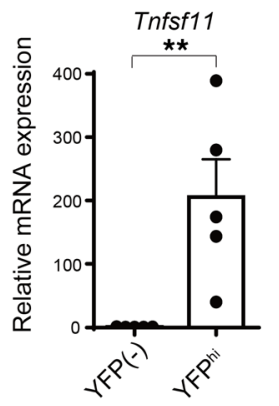

E

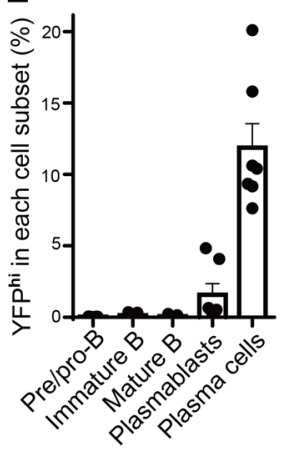

$\mathbf{F}$

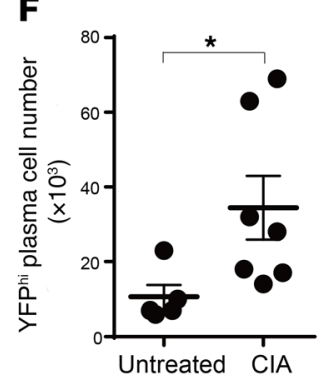

G

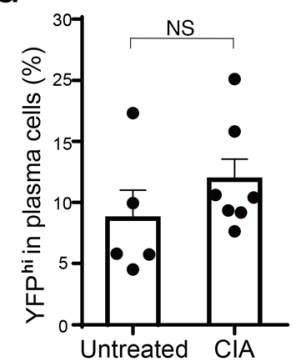

H

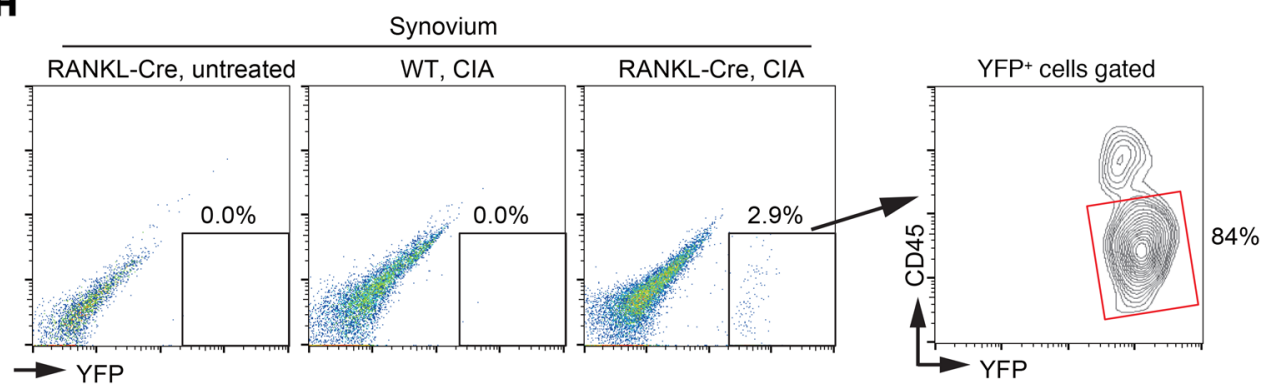

I

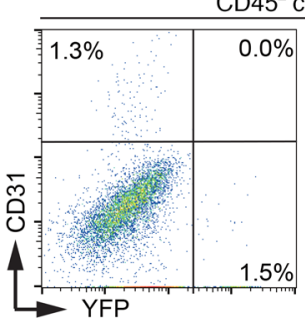

CD45- cells gated

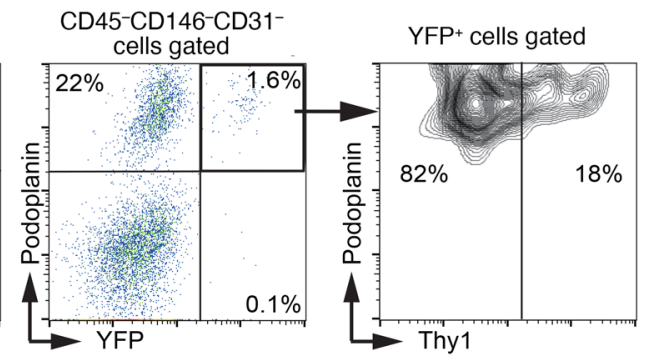

Figure 1. The number of RANKL-expressing plasma cells increases in the bone marrow under arthritic conditions. RANKL-Cre ROSA26-YFP mice with CIA were analyzed 3 weeks after the secondary immunization. The bone marrow (BM) of the tibias and femurs (A-G) and inflamed synovium (H and $\mathbf{I})$ were analyzed. (A) YFP expression in bone marrow cells. (B) The number of YFP'id (left) and YFP' (right) cells ( $n=5-7)$. (C) Tnfsf11 mRNA expression in YFP ${ }^{-}$and YFPh cells analyzed by qPCR $(n=5)$. (D) FACS profile of YFPhi cells. (E) The frequency of YFPhi cells in B-lineage cells in the bone marrow under arthritic conditions $(n=7)$. (F) The number of YFPhi plasma cells in the bone marrow $(n=5-7)$. (G) Frequency of YFPhi cells in the bone marrow plasma cells $(n=5-7)$. (H) YFP and CD45 expression in the synovium. (I) CD45- cells were examined for the expression of CD31, CD146, podoplanin, and Thy1. Representative data of 3 independent experiments are shown (A, D, and I). All data are expressed as the mean \pm SEM. ${ }^{*} P<0.05 ;{ }^{*} P<0.01$ by unpaired Student's $t$ test. NS, not significant. 
A

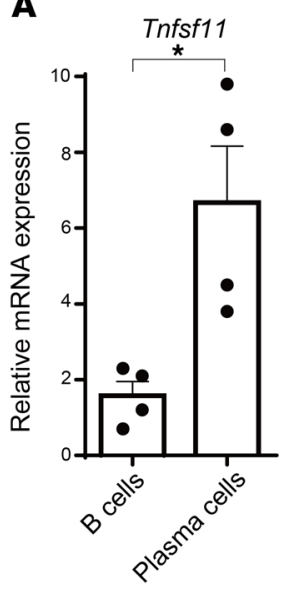

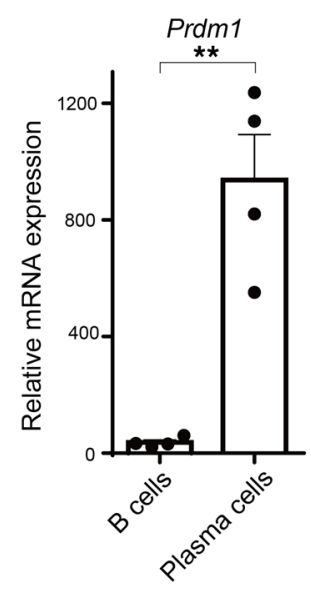

B

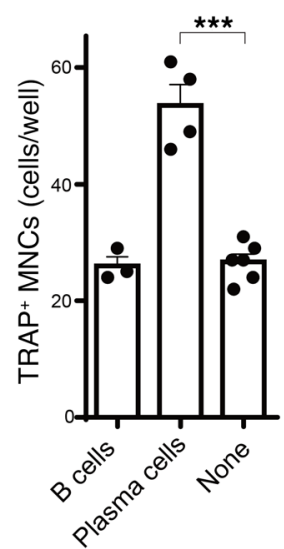

D

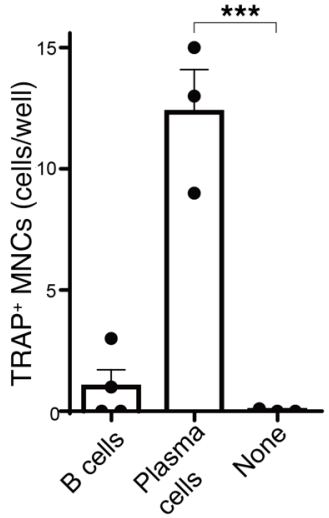

E

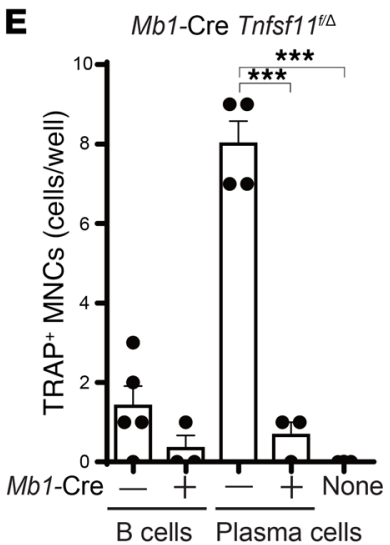

C

B cells

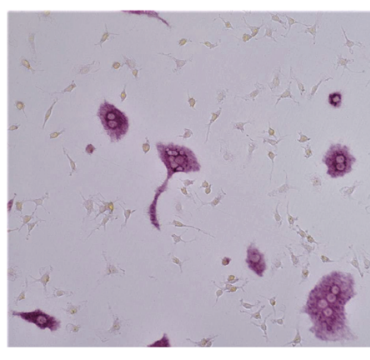

Plasma cells

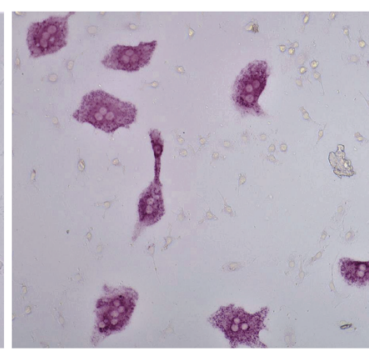

No B cells

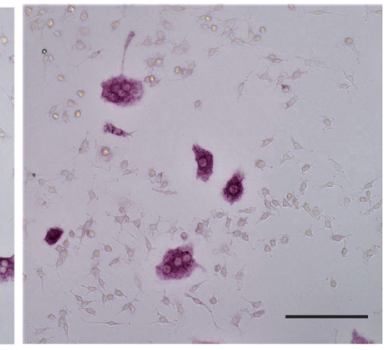

Pro/pre-B

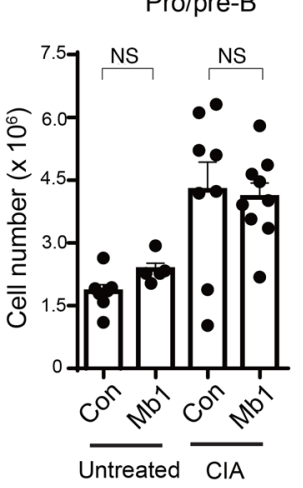

Immature B

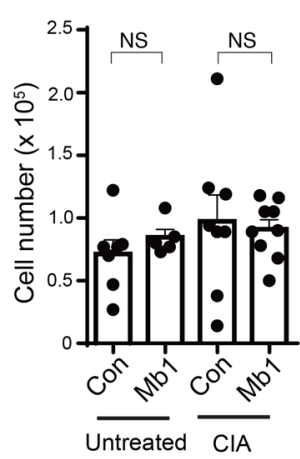

Mature B

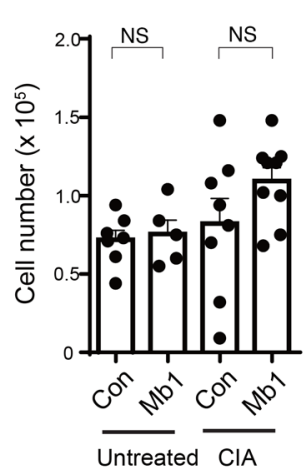

Plasmablast

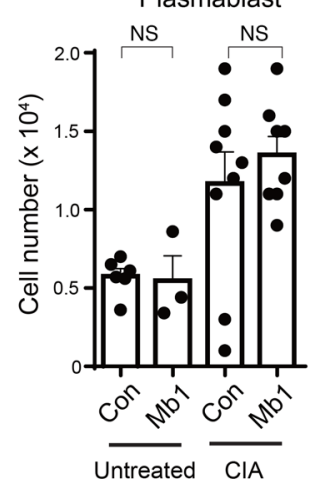

Plasma cell

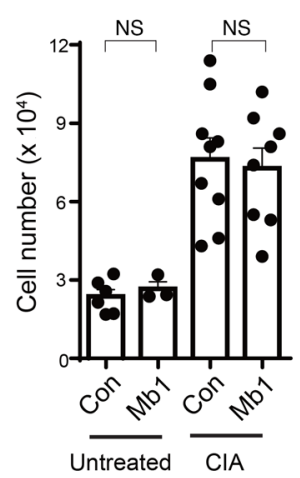

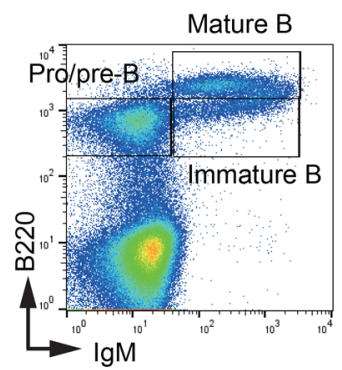

CD3-Gr1- cells gated

CD138+CD267+ cells gated
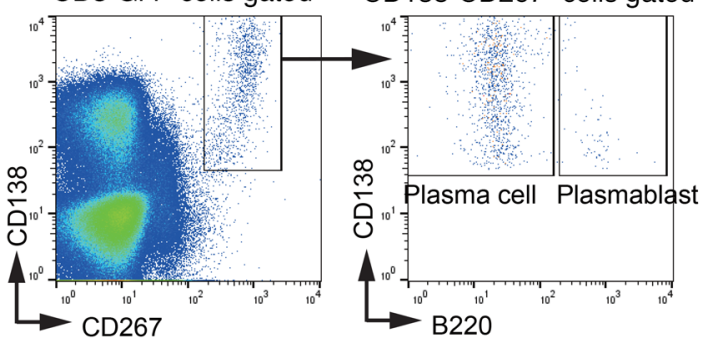

Figure 2. Plasma cells promote osteoclastogenesis in a RANIKL-dependent manner. Bone marrow plasma cells (CD3- $\mathrm{Gr1}{ }^{-} \mathrm{CD} 138^{+} \mathrm{B} 220^{-}$cells) or B cells (CD19+ ${ }^{+}$cells) were cocultured with osteoclast precursor cells for 5 days. (A) Tnfsf11 (left) and Prdm1 (encoding Blimp1) (right) mRNA expression of the bone marrow plasma cells and B cells before the coculture $(n=4)$. (B and C) Osteoclast formation after the coculture of osteoclast precursor cells derived from untreated DBA1/J mice with bone marrow plasma cells or B cells from arthritic DBA1/J mice $(n=3-6)$. The number of TRAP ${ }^{+}$multinucleated (more than 3 nuclei) cells (MNCs) is shown. Scale bar: $100 \mu \mathrm{m}$. (D and E) Osteoclast formation after the coculture of osteoclast precursor cells prepared from splenocytes

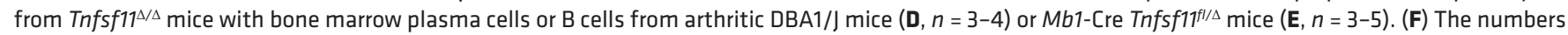

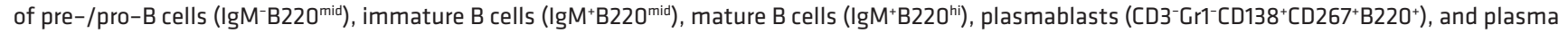
cells (CD3 $\left.{ }^{-} \mathrm{Cr1}{ }^{-} \mathrm{CD} 138^{+} \mathrm{CD} 267^{+} \mathrm{B} 220^{-}\right)$in the bone marrow under physiological and arthritic conditions $(n=5-9)$. All data are expressed as the mean \pm SEM. ${ }^{*} P<0.05$; ${ }^{* *} P<0.01 ;{ }^{* *} P<0.001$ by unpaired Student's $t$ test (A), 1-way ANOVA with the Holm-Sidak multiple-comparison test (B, D, and E), or 2-way ANOVA with the Holm-Sidak multiple-comparison test (F). NS, not significant. 
A

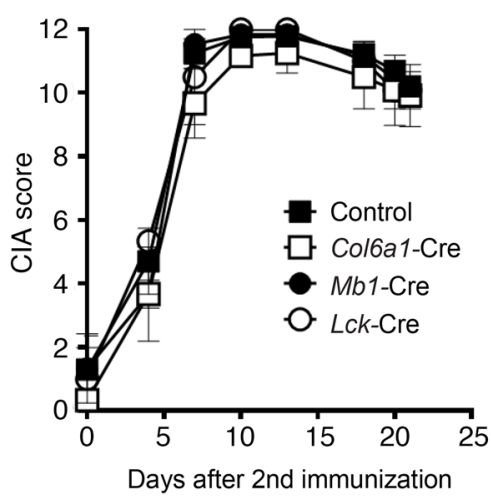

C

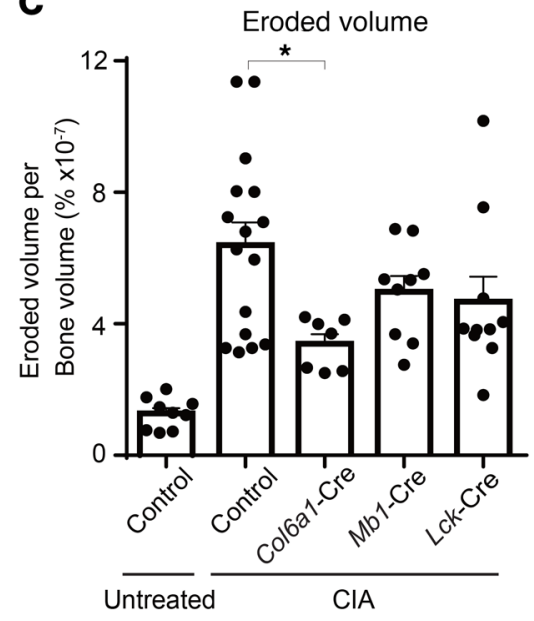

D

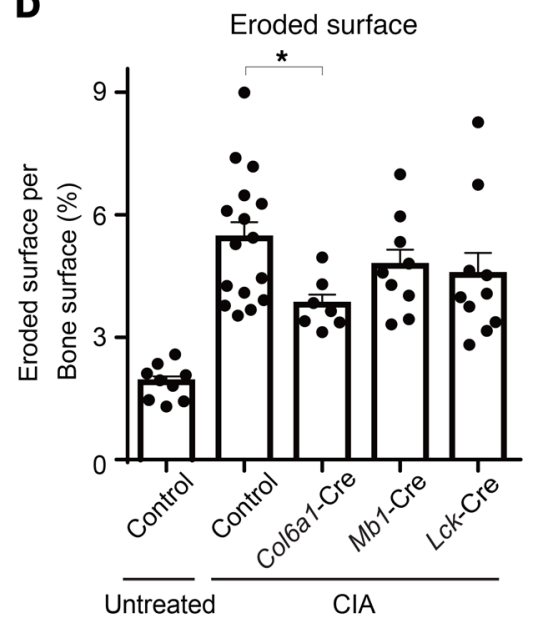

B

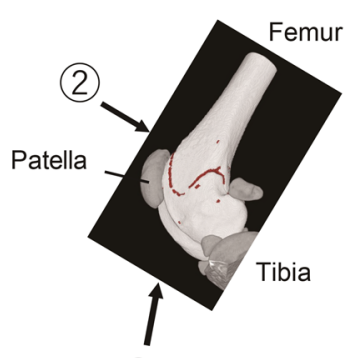

(1)
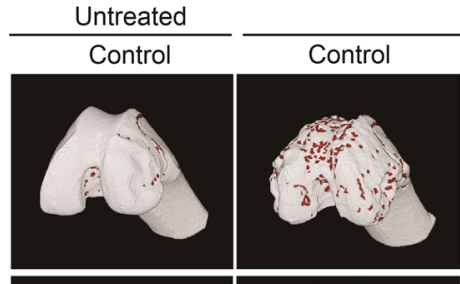

Col6a1-Cre

$\mathrm{CIA}$
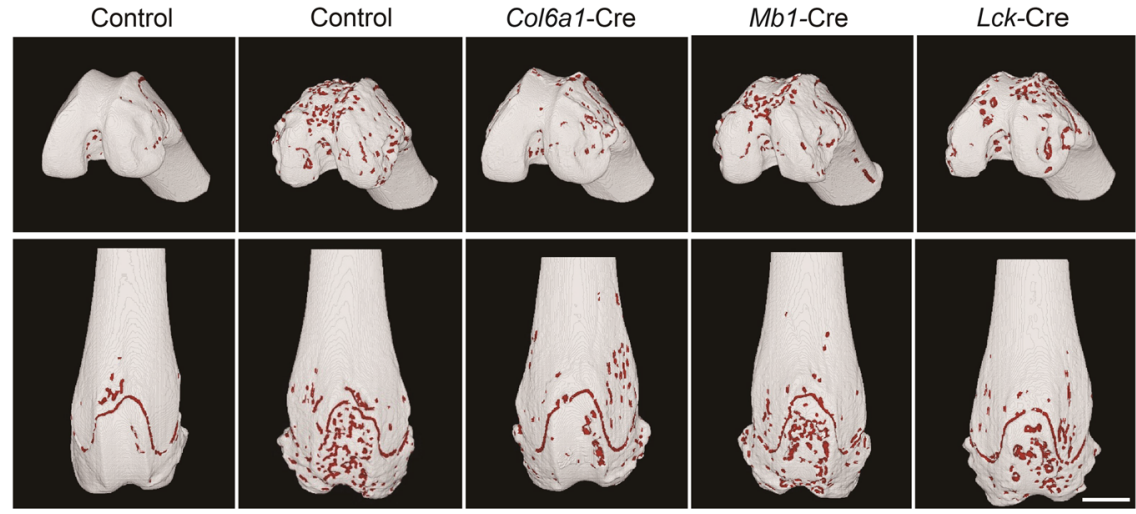

Figure 3. Synovial fibroblast RANKL, but not the RANKL derived from B-lineage cells, is responsible for joint destruction. (A) CIA score of Tnfsf17 fi/

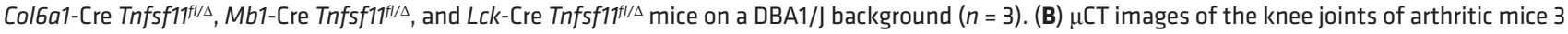
weeks after the secondary immunization. The red area shows cavities of femurs. Scale bar: $1 \mathrm{~mm}$. Representative data are shown. (C and $\mathbf{D})$ Eroded volume

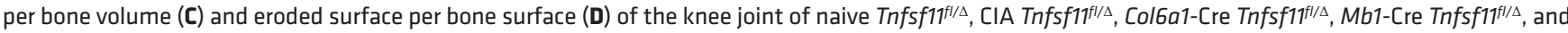
Lck-Cre Tnfsf17f/s mice $(n=7-16)$. All data are expressed as the mean \pm SEM. ${ }^{*} P<0.05$ by 1 -way ANOVA with the Holm-Sidak multiple-comparison test.

(Supplemental Figure 2). By crossing these mice with ROSA26loxP-Stop-loxP-YFP (ROSA26-YFP) reporter mice, we obtained a new RANKL-reporter mouse strain, RANKL-Cre ROSA26-YFP mice, in which YFP expression is turned on in the cells that are expressing RANKL or expressed RANKL.

We induced collagen-induced arthritis (CIA) in RANKL-Cre ROSA26-YFP mice and analyzed the YFP-expressing cells in the bone marrow and synovium. We noticed a marked increase in YFP ${ }^{\text {hi }}$ cell number in the bone marrow (Figure 1, A and B). Although YFP expression does not always correlate with mRNA expression in the fate mapping system, we confirmed that RANKL mRNA was highly expressed in YFPhi cells (Figure 1C). Flow cytometric analysis showed that the majority of YFPhi cells in the arthritic bone marrow were $\mathrm{CD}^{-} \mathrm{Gr1}^{-} \mathrm{CD} 267^{+} \mathrm{CD} 138^{+} \mathrm{B} 220^{-}$plasma cells (Figure 1D). The frequency of YFP ${ }^{\text {hi }}$ cells in plasma cells was the highest among the B-lineage cell subpopulations under arthritic conditions (Figure 1E). YFP plasma cells increased in number by approximately 3-fold under arthritic conditions, while the frequency of YFP $P^{\text {hi }}$ cells in the plasma cells was comparable between the physiological and arthritic conditions (Figure 1, F and G).
We next analyzed the inflamed synovium of RANKL-Cre ROSA26-YFP mice after induction of CIA. The majority of YFP $^{+}$ cells that appeared in the synovium only under arthritic conditions were negative for $\mathrm{CD} 45$ and positive for the fibroblast marker podoplanin (Figure 1, H and I). YFP was not detected in endothelial cells $\left(\mathrm{CD} 31^{+}\right)$or pericytes $\left(\mathrm{CD} 146^{+}\right)$(Figure 1I). The majority of YFP $^{\text {hi }}$ cells were negative for Thy1, suggesting that the RANKLexpressing bone-destructive synovial fibroblast subpopulation is enriched in Thy1 $1^{-}$rather than Thy $1^{+}$synovial fibroblasts (Figure 1I). Consistent with this, a recent single-cell analysis revealed that synovial fibroblasts in arthritis consist of fibroblast activation protein- $\alpha^{+} \mathrm{Thy}^{+}\left(\mathrm{FAP}^{+}{ }^{+} \mathrm{Thy} 1^{+}\right)$inflammatory and $\mathrm{FAP}^{+} \mathrm{Thy}^{-}$ bone-damaging subsets (20).

The high level of RANKL expression in bone marrow plasma cells in arthritis (Figure 2A) led us to examine whether plasma cells are able to promote osteoclast formation in vitro. B cells and plasma cells were purified from the bone marrow of arthritogenic DBA1/J mice after the induction of CIA. After the coculture of B-lineage cells with osteoclast precursor cells, we evaluated the formation of tartrate-resistant acid phosphatase ${ }^{+}$ 
A

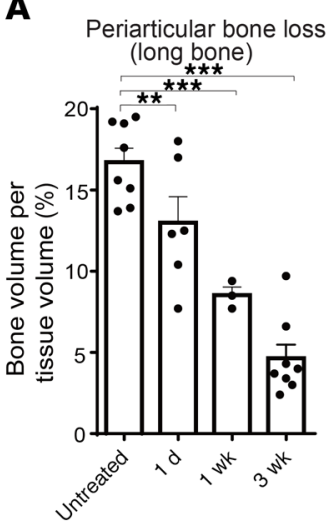

Systemic bone loss (spine)

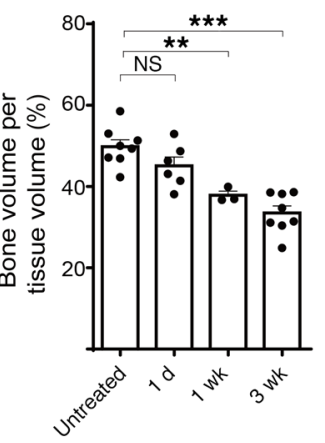

C
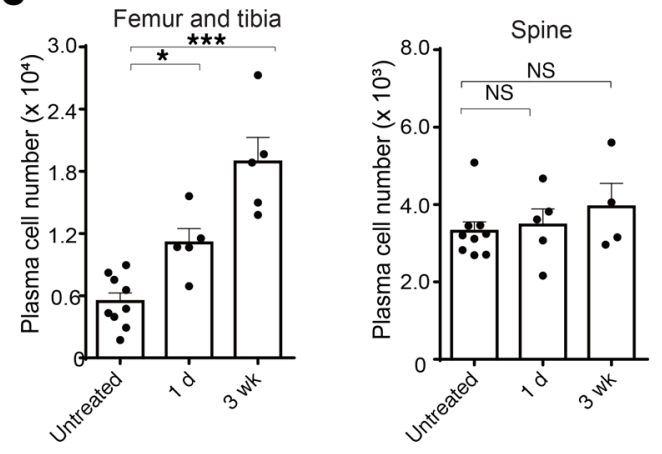

B

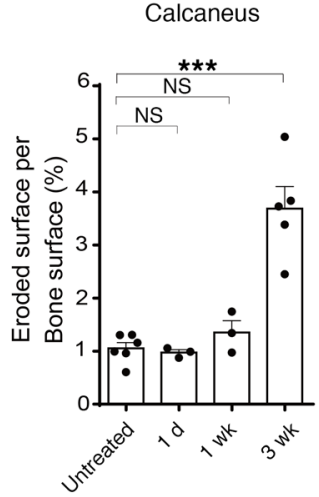

Calcaneus

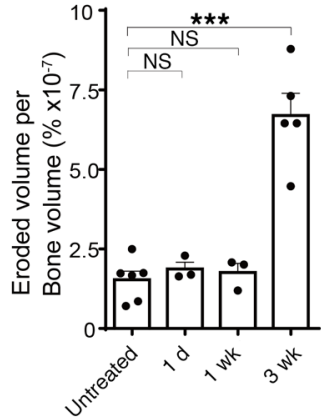

D

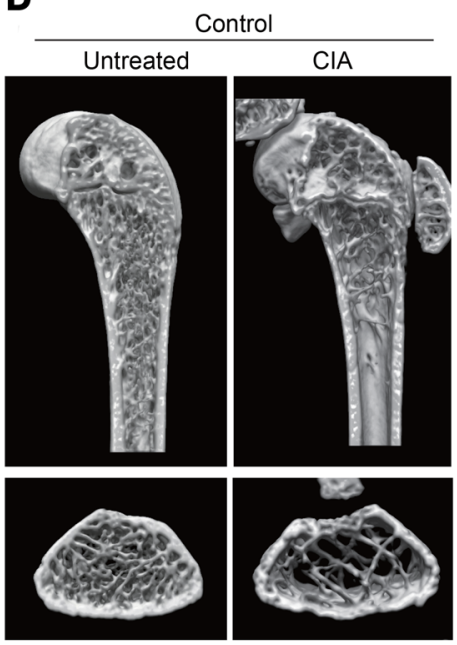

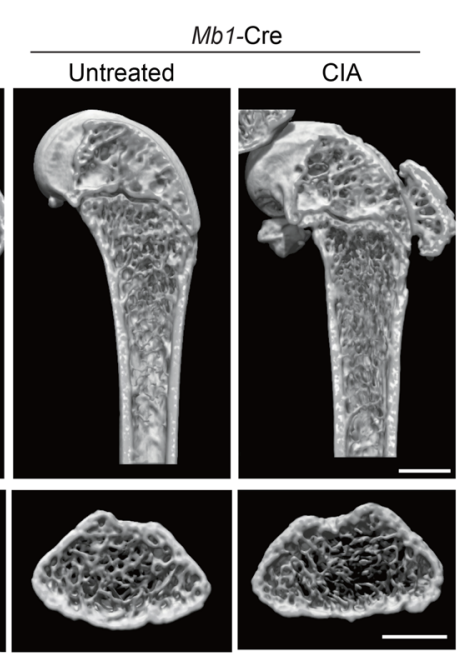

$\mathbf{E}$

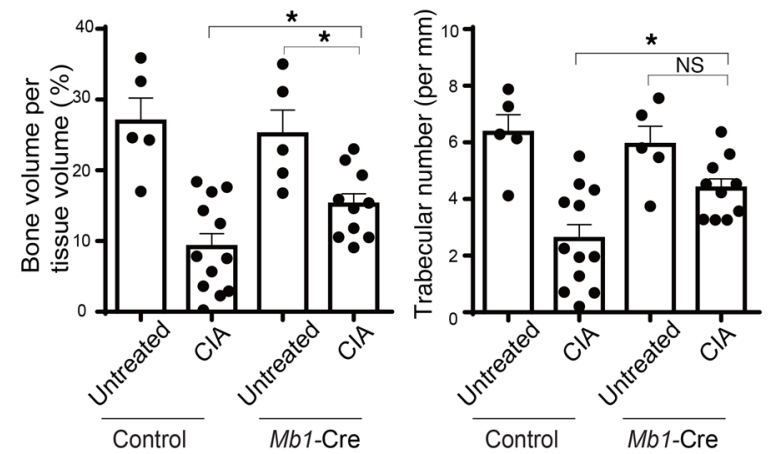

$\mathbf{F}$

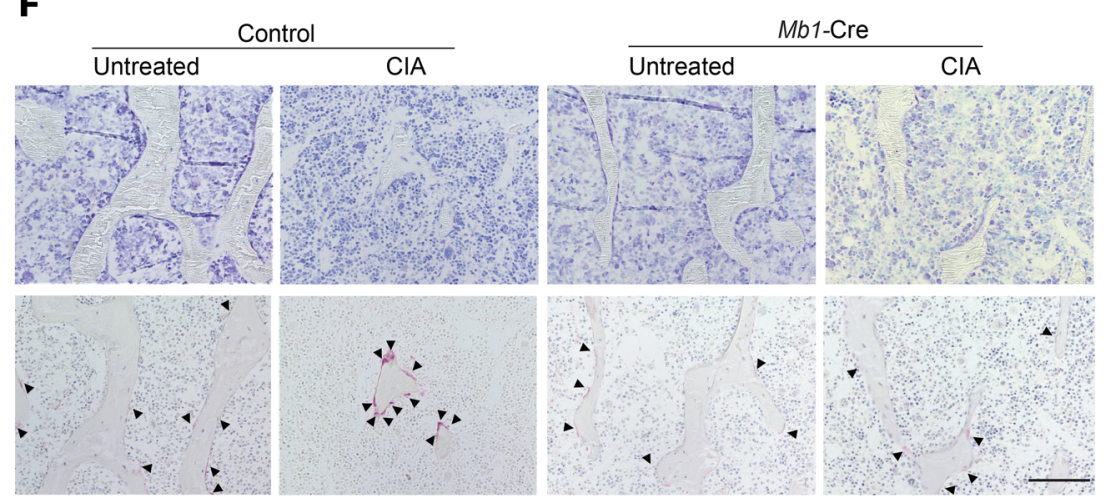

G

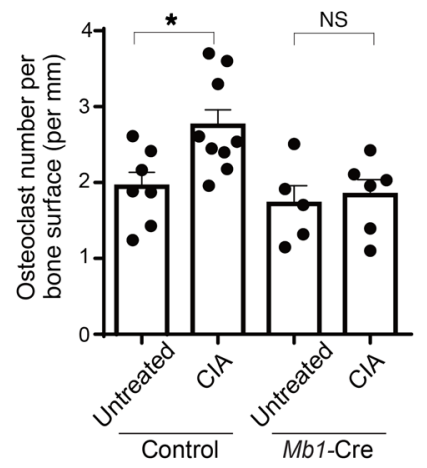


Figure 4. RANKL produced by B-lineage cells contributes to the periarticular bone loss in arthritis. (A and $\mathbf{B}$ ) Association of periarticular bone loss with an increase in the bone marrow plasma cell number. Bone volume per tissue volume of the distal femur (A, left, $n=3-8$ ) and lumbar spine (L5) (A, right, $n=3-8$ ) and joint erosion of the calcaneus bone (B, $n=3-6)$ during the course of CIA. (C) Number of plasma cells in femur and tibia (left) and lumber spine (L1-L3, right) during the course of CIA. (D) Representative $\mu \mathrm{CT}$ images of the distal femur of arthritic mice. Scale bars: $1 \mathrm{~mm}$. (E) Bone volume per tissue volume (left) and the trabecular bone number (right) of distal femurs of arthritic mice 3 weeks after the secondary immunization $(n=5-11)$. (F and $\mathbf{G})$ Representative $\mathrm{TRAP}^{+}$staining $(\mathbf{F})$ and the numbers of TRAP' multinucleated cells per bone surface $(\mathbf{G})$ of the tibia of arthritic mice $(n=5-9)$. Scale bar: $100 \mu \mathrm{m}$. All data are expressed as the mean $\pm \mathrm{SEM}$. ${ }^{*} P<0.05$; ${ }^{* *} P<0.01 ;{ }^{* *} P<0.001$ by 1 -way ANOVA with the Holm-Sidak multiple-comparison test (A-C) or 2-way ANOVA with the Holm-Sidak multiple-comparison test (E and $\mathbf{G}$ ). NS, not significant.

$\left(\mathrm{TRAP}^{+}\right)$multinucleated osteoclasts. In the absence of exogenous RANKL, the formation of osteoclasts was not observed. We then cocultured in the presence of a low concentration of RANKL and an antibody against osteoprotegerin (OPG), an inhibitor of osteoclastogenesis, because B cells are known to produce OPG. We found that the coculture with plasma cells but not with B cells enhanced the formation of bone-resorbing osteoclasts. This suggests that plasma cells rather than B cells promote osteoclastogenesis (Figure 2, B and C).

Does the RANKL expressed by plasma cells by itself induce osteoclastogenesis? RANKL expression in B-lineage cells is upregulated in the presence of $\mathrm{B}$ cell receptor (BCR)/CD40 stimulation and inflammatory cytokines, including $\operatorname{TNF}(21,22)$. However, TNF can also upregulate RANKL expression in stromal cells that contaminate osteoclast precursor cell cultures, which often hampers the interpretation of the results $(3,23)$. Thus, we obtained osteoclast precursors from RANKL-deficient mice, enabling us to eliminate the problem of an effect of RANKL from contaminating stromal cells (24). Furthermore, we added antibodies against OPG, IL-4, and IL-10 to neutralize osteoclastogenesis-inhibitory factors (3). Using this newly developed coculture system, we found that bone marrow plasma cells induce the formation of osteoclasts to a much greater extent than B cells (Figure 2D). Because this coculture system contains only RANKL-deficient cells except for plasma cells, the source of RANKL is evidently fully derived from plasma cells. These results clearly demonstrated that plasma cells promote osteoclastogenesis by expressing RANKL.

In order to demonstrate the role of RANKL expressed on plasma cells, we generated $M b 1$-Cre $T n f s f 11^{f / \Delta}$ mice, in which RANKL expression is specifically deleted in the B cell lineage (Supplemental Figure 3). While osteoclasts were formed by coculture with plasma cells derived from control $\operatorname{Tn} f s f 11^{t / \Delta}$ mice, the plasma cells from $M b 1$-Cre Tnfsf $11^{f / \Delta}$ mice failed to induce osteoclastogenesis. Thus, plasma cells induce osteoclastogenesis through RANKL expression (Figure 2E).

It was previously shown that global RANKL-knockout mice had a lower number of B cells, but the development of B cells was normal in mice specifically deficient in RANK in B cells (25, 26). B cell numbers in CD19-Cre $T n f s f 11^{f / f l}$ mice were reported to be slightly reduced (27). We found there was no difference in the number of B-lineage cells between $M b 1$-Cre $T n f s f 11^{f / \Delta}$ mice and the control mice under either physiological (Figure 2F and Supplemental Figure 4) or arthritic conditions (Figure 2F). The lack of RANKL in B-lineage cells did not affect the number of other hematopoietic cells (Supplemental Figure 5).

We next explored the function of RANKL expressed in $\mathrm{B}$-lineage cells in terms of joint erosion in comparison with that expressed on $\mathrm{T}$ cells and synovial fibroblasts using Mb1-Cre $\operatorname{Tn} f s f 11^{f / \Delta}, L c k$-Cre Tnfsf $11^{f / \Delta}$, and Col6a1-Cre Tnfsf1 $11^{f / \Delta}$ mice. All of the mouse strains were backcrossed onto the DBA1/J background in order to efficiently induce CIA. There was no difference in the onset rate or the severity of CIA among these strains (Figure 3A). Severe joint erosion was observed in the control $\operatorname{Tn} f s f 11^{f / \Delta}$ mice 3 weeks after the secondary immunization. Microcomputed tomography $(\mu \mathrm{CT})$ analysis of the knee joints revealed that bone erosion was reduced in Col6a1-Cre Tnfsf $11^{f / \Delta}$ mice, but not in Mb1-Cre Tnfsf $11^{f / \Delta}$ mice or Lck-Cre Tnfsf $11^{f / \Delta}$ mice (Figure 3, B-D). Therefore, we provide the first convincing evidence to our knowledge that it is the synovial fibroblast RANKL rather than the RANKL derived from $B$ or $T$ cells that is responsible for joint erosion, using mice on the arthritogenic DBA1/J background (7, 21, 22, 28, 29).

Given the increase in the $\mathrm{RANKL}^{+}$plasma cell number in the bone marrow, we hypothesize that plasma cells contribute to periarticular bone loss in autoimmune arthritis. We analyzed the distal femur of CIA DBA1/J mice by $\mu \mathrm{CT}$ and counted the number of $\mathrm{CD}^{-} \mathrm{Gr1}^{-} \mathrm{CD} 138^{+} \mathrm{CD} 267^{+} \mathrm{B} 220^{-}$bone marrow plasma cells during the course of CIA. The bone volume of the distal femur was decreased, while the number of bone marrow plasma cells was increased in arthritis (Figure 4, A and C). Importantly, even before the onset of arthritis, the bone volume of the distal femur was decreased and bone marrow plasma cell numbers were increased, as early as 1 day after the secondary immunization. In contrast, systemic bone loss in the spine and joint erosion occurred only after the onset of arthritis (Figure 4, A and B). In the spine, bone marrow plasma cell number did not increase during the course of CIA (Figure 4C). These results suggest a specific involvement of bone marrow plasma cells in periarticular bone loss.

In order to test the role of RANKL in B-lineage cells in the course of periarticular bone loss, we induced CIA in Mb1-Cre $\operatorname{Tn} f s f 11^{f / \Delta}$ mice and evaluated the bone volume of the distal femur at 3 weeks after the secondary immunization. Mb1-Cre $\operatorname{Tn} f f f 11^{f / \Delta}$ mice had a normal bone mass compared with the $\operatorname{Tn} f f f 11^{f / \Delta}$ mice under physiological conditions (Figure 4, D and E). Under arthritic conditions, the bone mass in the distal femur, as well as the trabecular bone number, was profoundly reduced in the control $T n f s f 11^{f / \Delta}$ mice, but the reduction was significantly ameliorated in $M b 1$-Cre Tnfsf $11^{f / \Delta}$ mice (Figure 4E). The number of osteoclasts was increased in the tibial metaphysis of the arthritic Tnfsf $11^{f / \Delta}$ mice (Figure 4, F and G). Importantly, the increase in the osteoclast number was abolished in arthritic Mb1Cre $\operatorname{Tn} f s f 1^{f / \Delta}$ mice, indicating the major contribution of B-lineage cell RANKL to osteoclast formation in periarticular bone loss. Despite no increase in osteoclast number in CIA Mb1-Cre $\operatorname{Tn} f s f 11^{f / \Delta}$ mice, the periarticular bone loss was not completely recovered by the lack of RANKL in B-lineage cells. It is possible that periarticular bone loss is also influenced by other factors such as increased osteoclast activity and decreased bone-form- 
ing activity. Given the major role of osteocyte RANKL in bone remodeling, it will be important to evaluate its contribution to periarticular bone loss in the future. The bone mass of the spine was reduced in the control $T n f s f 11^{f / \Delta}$ mice under arthritic conditions, but the reduction in bone mass was comparable between the control and $M b 1$-Cre Tnfsf $11^{f / \Delta}$ mice (Supplemental Figure 6). These results collectively indicate that RANKL in B-lineage cells is critically important for periarticular but not systemic bone loss in autoimmune arthritis.

This study provides a conclusive answer to the question of which cells are the main cellular RANKL source for periarticular bone loss and joint erosion in autoimmune arthritis using RANKLfloxed mice with an arthritogenic DBA1/J background. We showed that bone marrow plasma cells express RANKL and function as an osteoclastogenic cell subset in periarticular bone loss. In joint erosion, we clarified the greater contribution of synovial fibroblast RANKL over T cell or B cell RANKL.

Draining lymph nodes, the nearest lymph nodes to the inflammatory sites, are essential for the initiation and progression of immune responses. By analogy, the bone marrow in proximity to inflammatory joints may be called "draining" bone marrow where plasma cells reside and produce autoantibodies. We demonstrated that the plasma cell number in fact increased in the "draining" bone marrow near the inflammatory joints during arthritis. This is possibly because plasma cell survival factors such as IL-6, BAFF, and APRIL may be abundant in the "draining" bone marrow $(16,18)$. In the "draining" bone marrow, plasma cells provide both RANKL- and antibodymediated costimulatory signals that cooperate to powerfully promote osteoclastogenesis $(8,9)$. In addition, plasma cells can produce osteoclastogenic cytokines such as TNF and IL-17 (19). Thus, plasma cells robustly promote periarticular bone loss by producing RANKL as well as antibodies and inflammatory cytokines.

Anti-CD20 antibodies are known to be effective for controlling joint inflammation and erosion (1). Considering the lack of CD20 expression by plasma cells $(1,17,18)$, it is likely that anti-CD20 antibodies do not directly affect or deplete plasma cells, but rather, decrease plasma cells by depleting CD20expressing precursors. Directly targeting long-lived plasma cells residing in the bone marrow will lead to the development of a new strategy for inhibiting inflammation, as well as bone damage that occurs in arthritis, including periarticular bone loss.

\section{Methods}

The methods used are described in the Supplemental Methods section. Study approval. All experiments were performed with the approval of the Animal Ethics Committee of The University of Tokyo.

\section{Author contributions}

NK designed the study, performed experiments, interpreted results, and wrote the manuscript. SW, MY, NCNH, and MT performed experiments, interpreted results, and contributed to manuscript preparation. AT and WP performed experiments and contributed to data interpretation. SS, GK, and TN generated genetically modified mice, and contributed to data interpretation and manuscript preparation. HT directed the project and wrote the manuscript.

\section{Acknowledgments}

We are grateful to M. Reth (Max Planck Institute) for providing Mb1Cre mice. We thank T. Nitta, K. Okamoto, K. Matsuda, M. Komagamine, Y. Okada, L. Danks, T. Asano, K. Kusubata, Y. Ogiwara, and K. Kubo for thoughtful discussion and valuable assistance. This work was supported in part by a grant for the Grants-in-Aid for Specially Promoted Research (15H05703), Scientific Research B (18H02636), Challenging Research (18K19438, 20K21515), Young Scientists (19K18943) from the Japan Society for the Promotion of Science (JSPS), AMED under grant number JP20ek0410073, and AMED-CREST under grant number JP20gm1210008.

Address correspondence to: Hiroshi Takayanagi, 7-3-1, Hongo Bunkyo-ku, Tokyo 113-0033, Japan. Phone: 81.3.5841.3373; Email: takayana@m.u-tokyo.ac.jp.
1. McInnes IB, Schett G. The pathogenesis of rheumatoid arthritis. N Engl JMed. 2011;365(23):2205-2219.

2. Shim JH, et al. Bone loss in rheumatoid arthritis: basic mechanisms and clinical implications. Calcif Tissue Int. 2018;102(5):533-546.

3. Okamoto K, et al. Osteoimmunology: the conceptual framework unifying the immune and skeletal systems. Physiol Rev. 2017;97(4):1295-1349.

4. Sato K, et al. Th17 functions as an osteoclastogenic helper $\mathrm{T}$ cell subset that links T cell activation and bone destruction. J Exp Med. 2006;203(12):2673-2682.

5. Komatsu N, et al. Pathogenic conversion of Foxp $^{+} \mathrm{T}$ cells into TH17 cells in autoimmune arthritis. Nat Med. 2014;20(1):62-68.

6. Pettit AR, et al. TRANCE/RANKL knockout mice are protected from bone erosion in a serum transfer model of arthritis. Am J Pathol. 2001;159(5):1689-1699.

7. Danks L, et al. RANKL expressed on synovial fibroblasts is primarily responsible for bone erosions during joint inflammation. Ann Rheum
Dis. 2016;75(6):1187-1195.

8. Harre U, et al. Induction of osteoclastogenesis and bone loss by human autoantibodies against citrullinated vimentin. J Clin Invest. 2012;122(5):1791-1802.

9. Negishi-Koga T, et al. Immune complexes regulate bone metabolism through $\mathrm{FcR} \gamma$ signalling. Nat Commun. 2015;6:6637.

10. Ochi K, et al. Ten-year incidences of selfreported non-vertebral fractures in Japanese patients with rheumatoid arthritis: discrepancy between disease activity control and the incidence of non-vertebral fracture. Osteoporos Int. 2015;26(3):961-968.

11. Zerbini CAF. et al. Biologic therapies and bone loss in rheumatoid arthritis. Osteoporos Int. 2017;28(2):429-446.

12. Iwata $\mathrm{T}$, et al. Periarticular osteoporosis of the forearm correlated with joint destruction and functional impairment in patients with rheumatoid arthritis. Osteoporos Int. 2016;27(2):691-701.

13. Kleyer A, et al. Bone loss before the clinical onset of rheumatoid arthritis in subjects with antici- trullinated protein antibodies. Ann Rheum Dis. 2014;73(5):854-860.

14. van Schaardenburg D, et al. Bone metabolism is altered in preclinical rheumatoid arthritis. Ann Rheum Dis. 2011;70(6):1173-1174.

15. Engdahl C, et al. Periarticular bone loss in arthritis is induced by autoantibodies against citrullinated vimentin. J Bone Miner Res. 2017;32(8):1681-1691.

16. Goldring SR. Periarticular bone changes in rheumatoid arthritis: pathophysiological implications and clinical utility. Ann Rheum Dis. 2009;68(3):297-299.

17. Hiepe F, et al. Plasma cells as an innovative target in autoimmune disease with renal manifestations. Nat Rev Nephrol. 2016;12(4):232-240.

18. Lightman SM, et al. Survival of long-lived plasma cells (LLPC): piecing together the puzzle. Front Immunol. 2019;(10):965.

19. Pioli PD. Plasma cells, the next generation: beyond antibody secretion. Front Immunol. 2019;10:2768.

20. Croft AP, et al. Distinct fibroblast subsets drive 
inflammation and damage in arthritis. Nature. 2019;570(7760):246-251.

21. Meednu N, et al. Production of RANKL by memory B cells: a link between B cells and bone erosion in rheumatoid arthritis. Arthritis Rheumatol. 2016;68(4):805-816.

22. Ota Y, et al. Generation mechanism of RANKL(+) effector memory B cells: relevance to the pathogenesis of rheumatoid arthritis. Arthritis Res Ther. 2016;18:67.

23. Lam J, et al. TNF- $\alpha$ induces osteoclastogenesis by direct stimulation of macrophages exposed to permissive levels of RANK ligand. J Clin Invest. 2000;106(12):1481-1488.

24. Tsukasaki M, et al. LOX fails to substitute for RANKL in osteoclastogenesis. J Bone Miner Res. 2017;32(3):434-439.

25. Kong YY, et al. OPGL is a key regulator of osteoclastogenesis, lymphocyte development and lymph-node organogenesis. Nature. 1999;397(6717):315-323.

26. Perlot $\mathrm{T}$, et al. Development and function of murine B cells lacking RANK. J Immunol. 2012;188(3):1201-1205.
27. Onal M, et al. Receptor activator of nuclear factor $\kappa \mathrm{B}$ ligand (RANKL) protein expression by B lymphocytes contributes to ovariectomy-induced bone loss. J Biol Chem . 2012;287(35):29851-29860.

28. Kong YY, et al. Activated T cells regulate bone loss and joint destruction in adjuvant arthritis through osteoprotegerin ligand. Nature. 1999;402(6759):304-309.

29. Yeo L, et al. Cytokine mRNA profiling identifies B cells as a major source of RANKL in rheumatoid arthritis. Ann Rheum Dis. 2011;70(11):2022-2028. 